Einführung zum Thema

Unfallchirurg 2020 123 (Suppl 1):S1-S2

https://doi.org/10.1007/s00113-019-0690-0

(c) Springer Medizin Verlag $\mathrm{GmbH}$, ein Teil von Springer Nature 2019

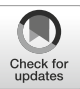

Marc Scheltinga - Percy van Eerten

Department of Surgery Máxima Medical Center, Veldhoven, The Netherlands

\section{Exercise-induced leg pain syndromes}

\section{The need for a multidisciplinary point of view}

Exercise-induced leg pain (ELP) may be caused by a range of distinct entities including chronic exertional compartment syndrome (CECS), medial tibial stress syndrome (MTSS), stress fractures, nerve entrapment syndromes and popliteal artery entrapment syndrome (PAES) [1, 2]. Historically these disorders are taught to us as separate and mutually exclusive, but the presence of multiple ELP syndromes with overlapping symptoms in one patient is not unusual. For instance, CECS of the deep flexor muscles (dp-CECS) as demonstrated by elevated muscle compartment pressures (ICP) is frequently combined with a painful distal tibial bone indicating concomitant MTSS. In addition, some patients who are eventually diagnosed with PAES have received earlier treatments for demonstrated CECS including fasciotomies. Focussing on just one ELP entity in an individual patient may lead to a monomaniac diagnostic approach and a disappointing outcome. It may therefore be argued that each ELP patient should undergo a standard workup including popliteal artery duplex scanning and ICP measurements to exclude the simultaneous presence of multiple ELP syndromes.

Type of CECS causing ELP is often determined by the type of sport. One nomogram calculated that a young male speed skater with bilateral anterolateral pain has a $90 \%$ chance of having antCECS. Conversely, soccer players with

The German version of this article can be found under https://doi.org/10.1007/s00113019-0689-6.
ELP tend to harbour dp-CECS [3]. CECS was previously thought to only occur in young athletes or military personnel. Interestingly, the incidence of CECS in general populations nowadays is increasing. A recent study in an ELP referral center found that one in eight individuals with CECS was a civilian over 50 years of age [4]. This trend of higher incidences of ELP in general populations is likely due to the 'exercise is medicine' movement that is currently embraced by large groups of active senior persons having sufficient spare leisure time.

A sound pathophysiological concept unifying ELP syndromes was never conceived. However, general terms such as 'overuse' and a 'mismatch between overload and recovery' are commonly used for the explanation of these pain syndromes $[5,6]$. It is likely that a combination of intrinsic (malalignments, joint instability, high foot arches) and extrinsic (insufficient technique, shoes, intensive sports) mechanisms contribute to onset and maintenance of ELP syndromes. Moreover, the presence of one ELP entity is a risk factor for a second one.

Patient's history and physical examination may both unveil clues that are pivotal in the diagnosis of an ELP syndrome. Pain and tightness lasting over hours following exercise are crucial for diagnosing CECS. Conversely, altered skin sensation as tested with a cotton swab may indicate a nerve-related ELP. Results of additional imaging and functional tests in this hypothetic patient may indicate normal nerve functioning erroneously suggesting that the pain is not related to nerve entrapment. In contrast, a stress fracture can only be diagnosed using an imaging technique, whereas history and physical examination are often nonspecific. The diagnostic road that an ELP patient travels is often long and winding as also reflected by an exceedingly long doctor's delay as documented in entrapment syndromes [7, 8]. A balanced diagnostic approach largely depends on an experienced multidisciplinary team as gold standard diagnostic tools are lacking in most ELP syndromes.

An ELP network ('NIAPS') that was recently initiated by Dutch surgeons and sports medicine physicians documented substantial regional differences in ELP management in the Netherlands. For instance, an operation for recalcitrant MTSS was standardly rejected by one research group but frequently performed by another. These heterogeneous and somewhat contradictory findings have urged NIAPS to form multidisciplinary teams including surgeons, sports medicine physicians, physiotherapists, neurologists and radiologists that are instructed to conceive protocols on diagnostic and therapeutic algorithms in ELP. One subgroup is currently working on defining standardization of patient outcome. A multidisciplinary approach is crucial to optimize management of future ELP populations.

The overall aim of a number of contributions in the current issue of Un- 
fallChirurg is to increase awareness in the domain of traumatologists and orthopedic surgeons regarding some of these ELP syndromes.
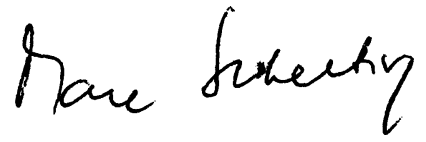

Dr. Marc Scheltinga, Veldhoven

\section{Corresponding address}

Dr. Marc Scheltinga
Department of Surgery
Máxima Medical Center
P.O. Box 7777, De Run 4600,
$\begin{aligned} & 5500 \text { MB Veldhoven, The } \\ & \text { Netherlands } \\ & \text { m.scheltinga@mmc.nl }\end{aligned}$

Conflict of interest M. Scheltinga and P. van Eerten declare that they have no competing interests.

The supplement containing this article is not sponsored by the industry.

\section{References}

1. Reinking MF (2007) Exercise related leg pain (ERLP): a review of the literature. N Am J Sports Phys Ther 2(3):170-180

2. Brukner P, Khan K, Bahr R (2016) Brukner \& Khan's Clinical Sports Medicine, 5th edn. McGraw-Hill, Sydney

3. de Bruijn JA, van Zantvoort APM, Winkes MB, van derCruijsen-Raaijmakers M, Hoogeveen AR, Teijink JAW, Scheltinga MRM (2018) Lower leg chronic exertional compartment syndrome in patients 50 years of age and older. Orthop J Sports Med 6(3):2325967118757179

4. de Bruijn JA, van Zantvoort APM, van Klaveren D, Winkes MB, van der Cruijsen-Raaijmakers M, Hoogeveen AR, Teijink JAW, Scheltinga MR (2018) Factors predicting lower leg chronic exertional compartment syndrome in a large population. Int $J$ Sports Med 39(1):58-66

5. Wilder RP, Magrum E (2010) Exertional compartment syndrome. Clin Sports Med 29(3):429-435

6. Franklyn-Miller A, Roberts $A$, Hulse D, Foster J (2014) Biomechanical overload syndrome: defining a new diagnosis. Br J Sports Med 48(6):415-416

7. van Zantvoort APM, Setz M, Hoogeveen AR, Scheltinga MR (2018) Common peroneal nerve entrapment in the differential diagnosis of chronic exertional compartment syndrome of the lateral lower leg: A report of 5 cases. Orthop J Sports Med 6(8):2325967118787761

8. Williams EH, Rosson GD, Hagan RR, Hashemi SS, Dellon AL (2012) Soleal sling syndrome (proximal tibial nerve compression): results of surgical decompression. Plast Reconstr Surg 129(2):454-462 